\title{
La prensa de Andalucía durante la transición
}

\author{
Manuel Ruiz Romero \\ Miembro del Grupo de Investigación en Estructura, Historia y Contenidos de la \\ Comunicación y colaborador honorario del Departamento de Historia Contemporánea \\ en la Facultad de Historia de la Universidad de Sevilla
}

Los medios de comunicación forman parte del entramado social que contribuyó a la democratización de España, especialmente entre las décadas de los años setenta ochenta. Considera el autor que al controlar los medios las instancias donde se ejerce el poder, la sociedad civil se encuentra más protegida y los propios medios consiguen liberarse del control político que les envuelve. Analiza las notas características de la transición andaluza, significando la no existencia de estudios sustanciales sobre la prensa en este periodo, que abarca desde las primeras elecciones a Cortes constituyentes (junio de 1976), hasta las primeras elecciones al Parlamento de Andalucía (mayo de 1982).

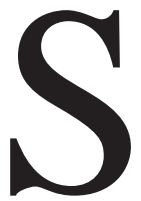

in insistir mucho en la polémica histórica acerca del comienzo de la transición, al no ser objeto directo del presente artículo, cabe comenzar este análisis afirmando que los medios de comunicación, sus profesionales, asociaciones del sector, y empresas promotoras son parte del entramado social, que hace inevitable el comienzo, evolución y buen fin del proceso en pro de la democratización en el que se ve inmerso este Estado, fundamentalmente entre las décadas de los años setenta ochenta.

Es más, si bien existen hitos en donde unos u otros autores enmarcan un comienzo simbólico de este proceso hacía la democracia en la vida pública, la transición sociológica, y con ella la influencia y el empuje de la transición periodística comienza mucho antes. Existe pues una clara diferenciación entre transición institucional y transición política. En la promoción de ese espíritu democratizador, aún desde el tardofranquismo y amparado en sus leyes como veremos, la prensa recupera un papel protagonista en la sociedad generador tanto de nuevas mentalidades como de actitudes. En la medida que los medios controlan a las instancias donde se ejerce poder, la sociedad civil se encuentra así más protegida, y en consecuencia, los propios medios consiguen li- 
berarse del control político que les envuelven. Culmina así la particular transición de la prensa ${ }^{1}$.

De otro lado, y antes de entrar en mayores análisis, conviene precisar metodológicamente algunas cuestiones, inmersas en la defensa que venimos realizando sobre la existencia de notas características en la transición andaluza $^{2}$. En primer lugar, no existen aún un sustancial elenco de estudios sobre la prensa en este periodo, de donde que realizar síntesis o valoraciones. Con salvedad de la obra ya clásica del profesor Checa Godoy, al realizar estas valoraciones partimos de limitaciones de las que no son ajenas nuestras instituciones, universidades o doctorandos, y en las que debemos citar honrosas excepciones ${ }^{3}$. La prensa andaluza se cita en demasía desde obras generales sobre la historia de la prensa española, una muestra histórica más, tal y como señala el citado profesor, de la subvaloración a que se encuentra sometida la prensa de nuestra Comunidad ${ }^{4}$. Al igual que mucho otros aspectos de la realidad científica, añadimos nosotros, de tal forma que esta constatación forma parte también de nuestro reto.

Por otro lado, y para situar al lector considero necesario al menos, sí limitar en el tiempo el concepto de transición andaluza. Tal y como ya he defendido, nuestro particular proceso, paralelo y complementario al realizado en todo el Estado, abarca desde las primeras elecciones a Cortes constituyentes (junio de 1976), hasta las primeras elecciones al Parlamento de

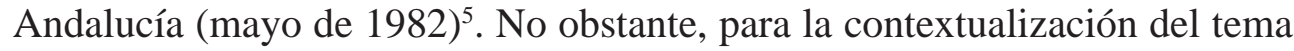
que analizamos, vamos a arrancar antes. Conscientes de que gran parte de la situación de la prensa andaluza durante la transición, se gesta -como en todo el Estado- años antes, y en el seno del propio régimen del general Franco. De igual forma, se puede afirmar que el final de la transición de la prensa

1 Así se expresa SINOVA, J., "La prensa en la transición a la democracia” en Cuenta y razón, (95), 1995, pp. $137-147$.

2 Cfr. de este autor: "Apuntes para una caracterización de la transición en Andalucía", en Revista de Historia Contemporánea, Sevilla, Universidad- Facultad de Historia, (8), 1998, (en prensa).

3 Destacar la tesis doctoral que en estos momentos finaliza sobre Diario de Cádiz, Aurora Labio Bernal compañera de este Grupo de Investigación, la cual es dirigida por Ramón Reig desde la Facultad de Ciencias de la Información de Sevilla.

4 Existen breves comentarios y reflexiones sobre los medios de la época en los volúmenes colectivos: Los Andaluces, Madrid, Istmo, 1.980; así como en Andalucía, Madrid, La Gaya Ciencia, 1978; y en Hacia una Andalucía Libre, Sevilla, Edisur, 1.978. Así como en cada una de las respectivas voces dentro de la Gran Enciclopedia de Andalucía.

5 Así lo justificamos en: "La transición de Andalucía. Fuentes, estado de la cuestión e hipótesis cronológica", en Actas del Tercer Congreso de historiadores sobre franquismo y transición, Sevilla, Universidad Pablo de OlavideCCOO, 1998 (en prensa). 
en Andalucía como quizás en otros puntos del Estado, no finaliza sino hasta 1984 por razones que nos disponemos a apuntar.

\section{Del reformismo a la democracia}

Desde la promulgación de la Ley de Prensa e Imprenta durante la primavera de 1966 comienza la formación de un denominado parlamento de papel, cuyos límites eran controlados por el Movimiento ${ }^{6}$. La prensa escrita pues, antes que otros ámbitos informativos, comienza aún bajo un discrecional y aleatorio régimen jurídico, un sistema de prácticas hasta ahora desconocidas. De tal forma que aún bajo el mismo margen legal, las cotas de libertad alcanzada en 1975 son objetivamente mayores que durante la fecha de su publicación oficial ${ }^{7}$.

Progresivamente las barreras a esta libertad periodística para el libre ejercicio de la pluralidad informativa van cayendo. Así la primera medida efectiva en este proceso de liberalización la puso el Decreto de 1 de abril de 1977, transcendente por cuanto suprimía el célebre y temido artículo dos de la Ley de 1966, el cual tanta represión había justificado contra los periodistas. Ese abril del mismo año se procede a la supresión del Ministerio de Información y Turismo, y a la conversión de la Prensa del Movimiento en el organismo Medios de Comunicación Social del Estado (MCSE) cuyas competencias se trasladan al ámbito de la cultura ministerial finalmente en julio de 1977 (Decreto 1558/1977). Por estos momentos, la disolución de la Cadena se anunciaba como próxima, a la vez que se anunciaba un futuro anteproyecto de Ley PRIVADO sobre el destino de sus 36 periódicos, 45 emisoras de radio y la Agencia Pyresa ${ }^{8}$.

La Constitución en orden jurídico, significa un punto de inflexión en el proceso de consolidación de las libertades y el derecho a comunicar $o$

\footnotetext{
6 Al respecto puede consultarse: FERNÁNDEZ AREAL, M. La Libertad de prensa en España 1938-1971, Madrid, Cuadernos para el Diálogo, 1971; DUEÑAS, G., La Ley de Prensa de Manuel Fraga, Madrid, Ruedo Ibérico, 1969; MOLINERO, C., La intervención del Estado en la prensa, Barcelona, Dopesa, 1971; así como ZALBIDEA, B., "Prensa del Movimiento: Los pasos contados hacia el aperturismo", en Historia de los medios de comunicación en España. Periodismo, imagen y publicidad (1900-1990), Barcelona, Ariel comunicación, 1989.

7 Un interesante análisis jurídico de esta época en estudio en: PASCUAL P.., "La prensa construyó la democracia”, en Historia de la transición y consolidación democrática en España, Madrid, UNED-UAM, tomo II, 1995, pp. 517 - 533. Con un interesante anexo de referencias legales, así como una recopilación anual de las ayudas estatales a la prensa desde 1976 a 1986.

8 EL PAÍS, 22-XII-1978. En esta época los incipientes comités de empresa comienzan a posicionarse ante la realidad de aquellas empresas de comunicación en la que trabajan. Tal es el caso de José Gutiérrez miembro del comité de empresa del diario $J A E ́ N$, el cual señala que esta cabecera puede llegar a ser democrática y rentable. Cfr. MUNDO OBRERO, 22-XII-1978.
} 
recibir libremente información veraz por cualquier medio de difusión. Abierto el debate sobre el futuro de los Medios de Comunicación Social del Estado, a partir de las primeras elecciones democráticas se abre un proceso parlamentario y gubernamental, en donde la voz de los trabajadores implicados se hace valer ${ }^{9}$. De la misma forma, la irrupción en el Congreso de los cinco diputados del Grupo andalucista, hace que se acentúe en Andalucía el debate sobre la transferencia de los medios escritos andaluces de la Cadena a la Junta preautonómica de Andalucía. Cuyo destino hasta esos momentos dentro del debate andaluz, había girado sobre la posible privatización o traspaso a sociedades de sus propios trabajadores.

Por último el desmantelamiento de la tutela informativa por parte del Estado tiene su punto y final en la subasta de los antiguos medios del Movimiento, realizada en mayo de 1984 por el primer gobierno socialista. Se daba así fin a una situación anacrónica dentro de un régimen democrático, cuyo control en manos de la coalición gubernamental de UCD, había vendo siendo objeto de críticas constantes por parte de los grupos de la oposición, especialmente por su utilización partidista en épocas electorales.

Medios andaluces de la Cadena de Prensa del Movimiento. Año $1965^{10}$

\begin{tabular}{lllll}
\hline \multicolumn{1}{c}{ Diario } & Localidad & Difusión & $\begin{array}{c}\text { Fecha } \\
\text { aparición }\end{array}$ & \multicolumn{1}{c}{ Director } \\
\hline Córdoba & Córdoba & 28500 & $25-7-41$ & Alvarez Gómez \\
Jaén & Jaén & 17000 & $01-4-41$ & Chamorro Lozano \\
La Tarde & Málaga & 14000 & $09-2-37$ & Villagordo Montalbán \\
La Voz de Almería Almería & 15000 & $01-4-39$ & Cirre Jiménez \\
La Voz del Sur & Jerez & 18000 & $01-7-36$ & Daroca de Val \\
Patria & Granada & 22000 & $09-2-35$ & Molina Fajardo \\
Sevilla & Sevilla & 24000 & $16-3-42$ & Arroyo Martín-E. \\
Sur & Málaga & 25000 & $10-02-37$ & Sanz Cagizas
\end{tabular}

\footnotetext{
9 Al respecto del inicio de este periodo puede consultarse: ZALBIDEA BENGOA, B., " La prensa del Movimiento en los primeros años de la Transición (1975-1979)”, en Historia de la transición y consolidación democrática en España, Madrid, UNED-UAM, tomo II, 1995, pp. 477 - 492.

${ }^{10}$ Anuario Prensa Española, 1965. No obstante, con anterioridad hay que señalar que desaparecen las siguientes cabeceras dentro de la cadena de Prensa del Movimiento: $F e$ en Sevilla, con aparición el 1 de septiembre de 1936 hasta el 16 de junio de 1946; Odiel en Huelva que se edita por primera vez en su segunda etapa el 1 de agosto de 1937; y Yugo en Almería, que comienza su salida el 20 de marzo de 1939.
} 
Bajo esta realidad, un informe interno realizado por la Prensa del Movimiento fechado en octubre de 1970 señala que el estado de las instalaciones es insostenible ${ }^{11}$. De las 35 instalaciones con las que cuenta la Prensa del Movimiento sólo se calificaba como aceptable de las establecidas en suelo andaluz a las que disponía La Voz del Sur (Jerez de la Frontera). En coherencia pues, se realiza un "Plan de Inversiones" destinado a la reconversión de las unidades de producción de la Cadena, el cual llegaba a superar los ochocientos cincuenta millones de pesetas, como inversiones previstas en dos fases entre los años 1972 y 1975.

En la primera se atiende aquellas cabeceras en donde resultaba especialmente urgente nuevas dotaciones y la adquisición de tecnología avanzada. Así de los veintidós medios designados ocho tienen implantación en alguna provincia andaluza. Un alto porcentaje (36\%) a los del resto del Estado, lo cual nos ofrece un dato objetivo sobre la dependencia de los medios escritos andaluces de gran parte de las provincias respecto a la citada Cadena, y por otro lado, a las necesarias inversiones en el sector provenientes del Estado. Los beneficiarios de esa intervención en Andalucía son los siguientes medios:

\begin{tabular}{ll}
\hline Diario & Inversión en millones \\
\hline Córdoba & 36,5 \\
Jaén & 25 \\
La Voz del Sur & 5 \\
Odiel & 25,5 \\
Sevilla & 27,5 \\
Sur y La Tarde & 54 \\
La Voz de Almería & $10,5^{12}$
\end{tabular}

Ese conjunto de circunstancias lleva a plantear en 1974 un "Plan de ajuste", para poner a estos medios, tal y como refleja documentos internos de la Delegación Nacional de Prensa y Radio del Movimiento, en condiciones de supervivencia y actualización como instrumento de orientación ideológica y política de la opinión pública española en tiempos que vivimos de

\footnotetext{
${ }^{11}$ Citado en: MONTABES PEREIRA, J., La prensa del Estado durante la transición política española, Madrid, CIS, 1989, pp. 40-41.

${ }^{12}$ De cara a la segunda fase citada se vuelve a beneficiar La Voz de Almería. Loc. cit.
} 
cambio y reforma. Este nuevo Plan, es presentado pues, como un segundo intento de reajuste. A partir de esa fecha se puede ya hablar en un sentido estricto de una Prensa estatal, sin dependencia teórica directa de organización política alguna ${ }^{13}$. Es decir, sin adscripción ideológica.

\section{Medios andaluces en la Cadena de Prensa del Movimiento. Año 1974}

\begin{tabular}{lcll}
\hline \multicolumn{1}{c}{ Diario } & Difusión & Viabilidad & Reestructuración de 1976 \\
\hline Córdoba & 9100 & Deficitario & Venta o alquiler \\
Jaén & 5981 & Deficitario & Venta o alquiler \\
La Tarde & 4707 & Deficitario & Cerrado en 1975 \\
La Voz de Almería & 6908 & Deficitario & Cerrado en 1975 \\
La Voz del Sur & 5781 & Deficitario & Venta o alquiler \\
Odiel & 5755 & Deficitario & Venta o alquiler \\
Sevilla & 4679 & Deficitario & Mantenimiento prioritario \\
Sur & 22659 & Rentable & Potenciar como rentable
\end{tabular}

Los primeros indicios de apertura en esta etapa que inicia su andar se van consolidando no sin esfuerzo contra el involucionismo. El Real Decreto Ley sobre Libertad de Expresión abre las puertas a una mayor amplitud y pluralidad informativa claramente democráticos, así como a la legalización de órganos internos de las fuerzas de oposición. Fundamentalmente, Mundo Obrero y El Socialista, cuyos militantes lo ofrecen por la vía pública o bien se ofrecen en kioscos y librerías como novedad editorial. Por cierto, que muchos de estos establecimientos de prensa fueron objeto de agresiones por parte de quienes deseaban mostrar así su rechazo a esta exposición pública de libertad.

La Constitución y la posterior liquidación de la cadena estatal de prensa Medios de Comunicación Social del Estado mediante acuerdo de Consejo de Ministros (16 de mayo de 1984), pone punto y final institucional a una amplia etapa de falta de libertades que en el medio informativo ha venido mostrando su cara entre la censura y la represión. En Andalucía de los once medios que configuran la citada Cadena en las ocho provincias para finales de 1978 sólo sobreviven siete cabeceras.

\footnotetext{
3 MONTABES PEREIRA, J., op. cit., p. 57.

${ }_{14}$ Ambos son cerrados en 1975 por Emilio Romero. Cfr. : MONTABES PEREIRA, J., La prensa del Estado durante..., op. cit. pp. $46-47$.
} 
Medios andaluces en el proceso de liquidación de prensa del Estado ${ }^{15}$

\begin{tabular}{llclc}
\hline \multicolumn{1}{c}{ Diario } & Localidad & $\begin{array}{c}\text { Difusión 1981 } \\
\text { (miles) }\end{array}$ & Pérdidas & $\begin{array}{c}\text { Precio subasta } \\
\text { en millones }\end{array}$ \\
\hline Córdoba & Córdoba & 9430 & Sí & 82,2 \\
Jaén & Jaén & 5660 & Sí & 60,3 \\
La Voz de Almería & Almería & 6160 & Sí & 93,4 \\
Odiel & Huelva & 5387 & Sí & 109 \\
Suroeste & Sevilla & 1645 & Sí & 94
\end{tabular}

Finalmente a lo largo de 1984 y a través de diferentes etapas, tienen lugar las distintas convocatorias de subastas de cada uno de los periódicos ${ }^{16}$. El 1 de febrero de 1984 son subastados, entre otras, las cabeceras de Córdoba y La Voz del Sur; el 21 de febrero: Odiel y Jaén; el 14 de marzo: Sur y La Voz de Almería.

El profesor Montabes Pereira como elaboración propia, y a partir de la información aparecida en prensa por esa fecha es quien ha elaborado cuadro sobre el resultado final del proceso de liquidación de la Cadena, de donde extraemos los titulares de medios andaluces ${ }^{17}$ :

\begin{tabular}{|c|c|c|c|c|c|c|}
\hline Diario & Difusión & Rentabilidad & $\begin{array}{l}\text { Precio } \\
\text { Subasta }\end{array}$ & $\begin{array}{c}\text { Precio } \\
\text { Adjudicación }^{18}\end{array}$ & $\begin{array}{c}\text { Empresa } \\
\text { compradora }\end{array}$ & $\begin{array}{l}\text { Tendencia } \\
\text { socio política }\end{array}$ \\
\hline Córdoba & 8524 & Deficitario & 67,8 & 82 & Norinfor, SA & $\mathrm{PSOE}^{19}$ \\
\hline Jaén & 5500 & Deficitario & 43,3 & 33,5 & Reinex, SA & PSOE \\
\hline La Voz de Al. & 5551 & Deficitario & 72,2 & 60 & Novotécnica & PSOE \\
\hline La Voz del Sur & - & Deficitario & 32,9 & - & 一 & - \\
\hline Sur & 25532 & Rentable & 526,8 & 526,8 & $\begin{array}{l}\text { Prensa } \\
\text { Malagueña }\end{array}$ & $\begin{array}{l}\text { Trabajadores } \\
\text { suscriptores }\end{array}$ \\
\hline \multicolumn{7}{|c|}{$\begin{array}{l}15 \text { Según BOE, } 21 \text { y } 22 \text { de octubre de } 1982 \text { (núm. } 252 \text { y 253). } \\
16 \text { Resolución del Consejo de Dirección del Organismo Público Autónomo Medios de Comunicación Social del } \\
\text { Estado. Publicado en BOE núm. } 307 \text {, de } 27 \text { de diciembre de } 1983 \text { y núm. 17, de } 20 \text { de enero de } 1984 . \\
17 \text { MONTABES PEREIRA, J., op. cit., p. } 152 \text { y } 153 . \\
18 \text { Ambos precios expresados en millones de pesetas. } \\
19 \text { Aunque el profesor Montabes cita en su cuadro a estas siglas de la organización socialista, entiéndase sectores } \\
\text { empresariales cercanos a esta fuerza política. }\end{array}$} \\
\hline
\end{tabular}




\section{Los medios de comunicación como sujetos y objeto}

La prensa no sólo es una fuente imprescindible para la temática que abordamos, sino que su estudio como documento histórico constituye un elemento de análisis esencial. La falta de fondos documentales de carácter oficial en Andalucía, viene siendo suplida por investigadores desde una especial atención a los medios escritos ${ }^{20}$. No obstante, conviene subrayar que progresivamente la información gestada desde la institución preautonómica, se abre espacio en la medida también, que la reforma política se asienta. Editoriales, secciones específicas, comentarios editoriales, entrevistas de fondo, reportajes, opiniones de firmas destacadas,... al paso del tiempo van consolidándose a la par del interés comunicativo y por ende público hacia la propia democracia y autonomía.

Pasemos a continuación a unos breves apuntes introductorios sobre los medios escritos en estos trascendentales años.

\section{A).- Las revistas}

Con anterioridad a la llegada jurídica de la libertad de expresión y como nota característica entendemos, de los medios impresos en la Andalucía de la transición, aparecen en nuestra Comunidad una serie de proyectos informativos surgidos a falta de una prensa democrática. Articulados en torno a grupos democráticos ven la luz en Andalucía un conjunto de nuevos títulos, al amparo de empresas o colectivos en donde se esconden las futuras elites de poder democráticas. Así, en septiembre de 1974 aparece en Sevilla La Ilustración Regional, que se presenta como Revista de Andalucía. Editada por la Sociedad Andaluza de Ediciones, S.A., tiene carácter mensual hasta su desaparición en enero de 1976. De gran calidad y amplia tirada, es víctima de la censura y entre sus promotores-colaboradores vamos a encontrar conocidos políticos y periodistas de la época, muchos de ellos activos hoy. Al hilo de esta desaparición aparece otro intento más modesto denominado Torneo: Semanario popular andaluz, el cual desde mayo de 1976 a principios de 1977, ejerce una limitada influencia alrededor de la provincia y como afirma Checa Godoy, se escora hacia posiciones cercanas al PSOE.

\footnotetext{
${ }^{20}$ Con esta técnica se ha realizado precisamente: HIJANO DEL RÍO, M. y RUIZ ROMERO, M. El Pacto Autonómico de Antequera (4 diciembre de 1978). Un documento para la historia de Andalucía, Sevilla, Consejería de Gobernación y Justicia-IAAP, 1997.
} 
Con carácter semanal y de forma más constante, aparece el autotitulado "Voz de Andalucía": Tierras del Sur. Con seguridad el intento más ambicioso, trascendente y decididamente difusor del regionalismo andaluz de la época. Dirigido por José María Javierre, se funda el 15 de mayo de 1976 y desaparece en 1979, editado en los talleres de El Correo de Andalucía. Precisamente de su proyecto empresarial y como editorial Argantonio, emerge arropado por el Congreso de Cultura Andaluza reunido en Archidona la denominada Enciclopedia de Andalucía, editada mediante fascículos ${ }^{21}$. Aquí aludida porque con seguridad es el proyecto editorial más ambicioso de nuestra Comunidad durante la transición, a la vez de una propuesta exitosa única en el tiempo. Intento de síntesis también pese a sus limitaciones, sobre la realidad pasada, presente y futura, que anuncia su aparición, junto a 1500 referencias bibliográficas sobre tema o autor andaluz. Motivando pues, al conocimiento de Andalucía, desde una perspectiva popular ${ }^{22}$. Una vez culmina el proyecto con el apoyo del primer gobierno preautonómico de Andalucía, con Alfonso Lazo como titular del departamento de Cultura, se presenta en Málaga, con salida al público a partir del 1 de marzo con cien fascículos. Un tratado sobre Andalucía en siete volúmenes, versando cultura y actualidad. Pendiente de actualización pero sin referentes en nuestra particular transición ${ }^{23}$. Ya en el acto se dice que la iniciativa representa el mayor y el más urgente servicio de carácter cultural que podemos realizar en favor del pueblo andaluz ${ }^{24}$.

Desde Málaga nace en febrero de 1979, al hilo de las primeras elecciones a Cortes Generales Algarabía, que como semanario apenas llega al año de vida ${ }^{25}$. De la misma forma que lo hace Naif en Almería, la cual no pasa de su tercer número, y que junto a Almería Semanal y Alborán conforman las iniciativas del oriente andaluz. Igualmente, y en su primera etapa,

21 ABC, 29-X-1978.

${ }^{22}$ SUR, 31-XII-1978. Al respecto de esta importante actividad editorial puede consultarse: MEDINA CASADO, M., "La Gran Enciclopedia de Andalucía", en Boletín del Centro de Estudios Históricos de Andalucía, (8), diciembre de 1996, pp. 4 - 6.

${ }^{23}$ Se presenta en el Palacio de la Madraza de Granada, y es editada por la institución Cultura Viva, el semanario Tierras del Sur, y la editorial Anel. Cfr. IDEAL, 18-II-1979. Su presentación en Málaga en SUR, 9-III-1979.

${ }^{24}$ PATRIA, 21-II-1979. Sus dos primeros fascículos son presentados más tarde en Sevilla. EL CORREO DE ANDALUCÍA, 25-II-1979; HOJA DEL LUNES, 26-II-1979 y EL PAÍS, 28-II-1979. De tal forma que el inicio de su comercialización se anuncia para la última semana del mes de marzo.

${ }^{25}$ REIG GARCÍA, R., «Las revistas andaluzas en la transición (1974-1979) y el caso de `Algarabía`, en Revista de Estudios Andaluces, (17), 1991, pp. 63-83, en donde puede verse una introducción de carácter general de las revistas de la transición andaluza. En la presentación del número cero se define al nuevo semanario como andaluz para Andalucía. Cfr. la presentación en Málaga en SUR y SOL DE ESPAÑA, 7-II-1979; así como en Sevilla NUEVA ANDALUCÍA, 2-III-1979. 
aparece desde Sevilla la revista Leviatán, cuyo consejo de dirección ofrece clara idea de la cercanía ideológica al PSOE andaluz ${ }^{26}$.

En orden a las revistas de carácter cultural, las instituciones democráticas han acentuado y consolidado hasta nuestros días, estas publicaciones fundamentalmente alrededor de Universidades, la propias instituciones de autogobierno andaluz, Diputaciones y corporaciones locales ${ }^{27}$. Con carácter preautonómica en referencia al espacio de tiempo en estudio, cabe destacar también por su independencia y permanencia en el tiempo Candil editada por la Peña Flamenca de Jaén desde $1978^{28}$. De la misma forma, la malacitana Jábega, la cual con carácter trimestral ha venido desde el tardofranquismo ejerciendo su influencia, potenciada con absoluta normalidad con la llegada de la nuevas corporaciones provinciales democráticas.

\section{B).- Diarios}

Tras un periodo de obligado inmovilismo, la prensa andaluza de la transición debe resultar caracterizada por su abierta inestabilidad. Distintos títulos aparecen en las provincias andaluzas, por un lado, como consecuencia de la democratización de los antiguos pertenecientes a la Cadena, por otro, como fruto de nuevas experiencias editoriales ${ }^{29}$.

Si bien el carácter vespertino se cierra en 1975 con La Tarde de Málaga, reaparece en junio de 1976 bajo la experiencia de Nueva Andalucía nacido tras el fracaso de Sevilla y que sucumbe finalmente a finales de 1984 sin más implantación que la conurbación hispalense y destacando por su abierto andalucismo. Editada en los talleres gráficos de El Correo de Andalucía, autotitulado como Diario Católico desde que en 1899 lo fundara el cardenal hispalense Marcelo Spínola. Etapa tras la que el medio entra en una crisis

\footnotetext{
${ }^{26}$ Antonio Guerra su Director, con Alfonso Guerra, José Luis López López y José Rodríguez de la Borbolla. Cfr. Sección El politicón en SUROESTE, 26-XII-1978.

${ }^{27}$ Cfr. ESCOBAR PÉREZ, B., "Publicaciones periódicas de la Administración Andaluza", en Sistemas y políticas de información en el Estado de las Autonomías. I Jornadas Andaluzas de Documentación, Sevilla, Asociación Andaluza de Documentalistas, 1997, pp. 165-174..

${ }^{28}$ Al respecto de la prensa flamenca puede consultarse: CHECA GODOY, A., "La prensa flamenca. Acercamiento a un modelo en expansión", en Actas del VII Congreso sobre el Andalucismo Histórico, Sevilla, Fundación Blas Infante, 1997, pp. $693-702$.

${ }^{29}$ De manera aislada un único ensayo sobre la prensa andaluza de la transición: PABLOS, MERCEDES DE; $L a$ prensa, Sevilla, Grupo Andaluz de Ediciones, 1.981. En referencia a la temática prensa y transición hispalense puede consultarse la ponencia del autor: «Prensa hispalense y transición andaluza. Balance y perspectivas.» en el Ciclo La prensa vista por la prensa. Aproximación al periodismo hispalense contemporáneo organizado durante el mes de noviembre de 1997, por el Departamento de Comunicación, dentro de la Facultad de Ciencias de la Información de la Universidad de Sevilla (en prensa).
} 
que culmina con la pérdida paulatina de peso de los sectores eclesiásticos dentro del Consejo de Administración, a favor de sectores afines del partido en esos momentos hegemónico en el poder: PSOE. Y es que el intento empresarial por consolidar un medio para la tarde andaluza se apunta como nota característica, mientras se recoge la experiencia de fracasos en este sentido. Así, Sevilla, como diario del Movimiento se transforma en matutino Suroeste, concebido bajo el ambicioso carácter de Diario Regional de Andalucía. Proyecto que no cuaja y en la medida que transcurren los años desde su aparición en junio de 1976, desaparece finalmente en febrero de $1983^{30}$.

Posiblemente motivado por este contexto de nuevos intentos empresariales desde la órbita de $\mathrm{ABC}$ edición de Sevilla, va a aparecer el vespertino Informaciones de Andalucía. Nueva intentona de diario regional para Andalucía, la cual editada desde los talleres de la solvente edición hispalense de $A B C$, y a tenor de su limitada tirada, resulta ser más trascendente por su iniciativa empresarial que por su capacidad de influencia periodística. Ello pese al apoyo que se le realiza desde la cabecera de donde parte su existencia $^{31}$.

Junto a ellos y a tenor de nuestra experiencia, ABC en su edición de Sevilla, superando planteamientos sucursalistas de Madrid, se convierte bajo la dirección de Nicolás Salas y a través de las primeras secciones que existen sobre información regional o en referencia al ente preautonómico; se convierte como decimos, en una imprescindible fuente de información para el estudio de la transición en nuestra Comunidad ${ }^{32}$. Y ello gracias a su amplio despliegue para informar y evaluar las noticias de ámbito andaluz en el desarrollo democrático/autonómico. Gracias también a su excelente tirada, que le hace superar en rentabilidad a la edición madrileña, y al serio intento de liderar la información regional a partir de un sustancial aumento de páginas dedicadas a este tipo de información ${ }^{33}$.

${ }^{30}$ Véase junto a la obra antes citada del mismo autor: CHECA GODOY, A. Historia de la prensa giennense 1.8081.983, Jaén, Diputación, 1.986.

${ }^{31}$ El buen éxito de este medio provoca rumores que presentan a Nicolás Salas como director de la MCSE Sección El Politicón. EL CORREO DE ANDALUCÍA, 8-IV-1979.

${ }^{32}$ Esta dimensión puede observarse bien, aún desde una óptica parcial al centrarse el estudio sólo en aspectos versados con la capital hispalense en: HUERTA MARTÍNEZ, A., Sevilla en su diario ABC, Universidad de Sevilla, tesina de licenciatura inédita, 1985.

33 Va a ser e Ortiz de Lanzagorta quien inicie en las páginas de ABC días después de la toma de posesión de Nicolás Salas como nuevo director de esta cabecera en su edición hispalense, una nueva sección denominada bajo el blasinfantiano título de Ideal andaluz. Con una aparición casi diaria ven la luz diferentes artículos y comentarios sobre temas de actualidad sevillanos y andaluces, va a ir, y estos es lo que nos interesa subrayar, apareciendo por primera vez distintos contenidos sobre la vida y obra de Blas Infante, los denominados andalucistas 
Por último, siempre dentro de la limitación cronológica ya apuntada como final de la transición de Andalucía (formación del Parlamento de Andalucía en 1982), debemos señalar en Córdoba la aparición en mayo de 1981 del diario La Voz. Surgido desde promotores respaldados también por el PSOE, resulta cerrado en 1984 en la medida que su capital se hace con la propiedad por subasta del Córdoba. De igual forma, debemos citar en el periodo en estudio Sol de España que aparece desde Marbella, más tarde desde Málaga; Patria en Granada como antiguo diario falangista; así como Diario de Cádiz como una de las experiencias más rentables, junto a Area que trasciende en el tiempo a partir de la su nacimiento como prensa local en La Línea de la Concepción. La provincia de Cádiz pues se consolida como la de mayor número de cabeceras durante este periodo.

En referencia a la prensa de partidos cabe destacar las referencias que pudieran existir en Mundo Obrero, Nuestra Bandera y El Socialista. Las cuales pese a su perspectiva de Estado, y dada la alta implantación de estas fuerzas en nuestra Comunidad, prestan un especial seguimiento a la cuestión andaluza, amén de otras publicaciones o boletines de carácter local/provincial. Junto a ellas Andalucía Libre, órgano del PSA que conoce varias etapas, y La Voz del Pueblo Andaluz editado por el PTA más tarde PAU-PTA ${ }^{34}$. A su vez, Andalucía Socialista, como portavoz de la FSA-PSOE que irá desapareciendo progresivamente en favor de potenciar El Socialista como prensa del Estado.

En el terreno sindical destacar Realidad de CCOO de Andalucía, Voz Obrera de las Comisiones obreras malacitanas, Tierra y Libertad órgano del SOC; otros de ámbito local de UGT así como Andalucía libertaria de CNT. En un intento de evaluar los sucesos de la transición se pueden apuntar también algunos interesantes artículos en la independiente Nación Andaluza ${ }^{35}$.

históricos, así como distintos comentarios ensalzando símbolos, recuperando o relacionando hitos del pasado autonomista hasta ahora relegado, menospreciado o perseguidos. Los pormenores del hecho y sus consecuencias, a la vez de la influencia procurada por el andalucista histórico Alfonso Lasso de la Vega, puede consultarse en el prólogo de Nicolás Salas en: HIJANO DELRÍO, M. y RUIZ ROMERO, M.; Sevilla en el recuerdo. Alfonso Lasso de la Vega, una vida dedicada a la cultura andaluza, Sevilla, Centro de Estudios Históricos de Andalucía, 1995, pp. 11 a 20. De la misma forma, la revista Mundo editada en Barcelona, en su número 1838 del día 26 de julio de 1975 reproduce el hecho a la vez que en su páginas interiores despliega un especial sobre Andalucía que sirve de titular a la portada: Poder Andaluz. ABC reproduce en portada fotografía del primer izado de la bandera verde blanca y verde en la feria de Muestras Iberoamericana de Sevilla. ABC, 28-I-1977.

${ }^{34}$ Durante la campaña a las elecciones generales de 1979 se anuncia la presentación del primer número del periódico del PTA, que se dice, posee una tirada de 50.000 ejemplares. Cfr. EL CORREO DE ANDALUCÍA, 7-II-1979.

35 VOZ OBRERA, Cuatro años de historia del movimiento obrero en Málaga, Málaga, Bandera Roja, 1978. 
En definitiva, un amplio espacio temático de investigación específica, aún por cultivar, y a desarrollar de forma paralela al estudio del resto de los medios de comunicación andaluces de la época ${ }^{36}$. Dicho de manera más clara: existe una bibliografía al respecto muy genérica y a veces útil por datos puntuales y referencias para el estudio de la prensa a la llegada de la democracia, pero estamos a la espera aún, de resultados y enfoques propios sobre los medios andaluces en el franquismo y la transición a la democracia ${ }^{37}$.

En cualquier caso y pese a las responsabilidades antes citada en la introducción del presente artículo, la prensa andaluza como parte importante del patrimonio documental andaluz se encuentra aún pendiente de una decidida acción institucional por parte de la Junta de Andalucía, la cual debe entenderse como respaldo definitivo al proveniente desde otros campos Fundamentalmente por lo que respecta a la conservación, defensa y difusión de una parte importante de nuestra historia de ayer y hoy ${ }^{38}$.

\section{Transición política de Andalucía y prensa}

Asistimos a un fenómeno general a todo el periodo, que tiene también su reflejo en la sociedad andaluza: la aparición de un tipo de prensa con diferentes ritmos de edición, relacionada abiertamente con opciones políticas. Característica ésta, que sin bien irrumpe con fuerza como en el resto del Estado, no llega a consolidarse finalmente tal y como ocurre en otros puntos. Particularmente en el País Vasco para los casos de Deia y Egín como importantes referentes de prensa diaria aún hoy ${ }^{39}$.

Todos estos fenómenos que aquí apuntamos van a constituir un marco caracterizado por un rápido ritmo de permanentes transformaciones (técni-

\footnotetext{
${ }^{36}$ Los fondos correspondientes a los Medios de Comunicación Social del Estado, se encuentra dentro de la Sección Ministerio de Cultura depositados en el Archivo General de la Administración de Alcalá de Henares, en sugerente invitación al estudio de los medios andaluces de le época.

37 Al respecto: BELINCHON BELINCHÓN, M., «Los medios de comunicación de masas como fuente para la historia contemporánea», en Historia Contemporánea y Nuevas Fuentes, Alicante, Universidad, 1.995.

${ }_{38}$ En el Anexo puede consultarse el texto del acuerdo plenario del Parlamento de Andalucía a favor de la creación de una Hemeroteca General de Andalucía (BOPA núm. 78, 10 mayo 1995, p 4406). No obstante al respecto: COBACHO MÁRQUEZ, M., ¿Quién tema a la Hemeroteca de Andalucía?, EL CORREO DE ANDALUCÍA, 31-XII-1997; así como DARBÓN, FERNANDO; Hemeroteca de Andalucía: ¿Una cuestión de semántica? EL CORREO DE ANDALUCÍA, 16-II-1998. La Asociación de la Prensa de Granada ha solicitado también su puesta en marcha por acuerdo de su Junta Directiva. Cfr. EL PAÍS, 4-XII-1997.

${ }^{39}$ Como ejemplos significativos del avance de estos estudios en otras comunidades autónomas cabe citar: LÓPEZ PÉREZ, S. La Prensa diaria de la Comunidad Autónoma del País Vasco durante la transición y la democracia (1975-1982), TDI, Universidad del País Vasco, 1992; así como CAMINOS MARCET, J.Ma ., Transformación de la prensa en Guipúzcoa durante la transición política. La Voz de Euskadi: nacimiento y fracaso de un proyecto informativo, Universidad del País Vasco, 1993.
} 
cas y empresariales) que van a operar en una etapa política de extremo interés social. Cambios que también afectan a los medios de comunicación escritos, y a todo lo que conlleva el mundo periodístico editorial y su consecuente estrategia informativa la cual pasamos a analizar ${ }^{40}$.

Por otra parte, otro importante factor de transformación lo supone el empuje y el protagonismo adquirido por las nuevas generaciones de profesionales de la información. Y no sólo por lo que significa el transcurrir del tiempo como ritmo biológico, sino por la progresiva implantación y reconocimiento de las Facultades de Ciencias de la Información ${ }^{41}$.

La transición como cambio de las estructuras políticas y sociales lleva pareja en los medios impresos unos cambios generalizados en todo el mundo occidental, que dan origen a una época considerada como nueva y diversamente definida como posmoderna, postindustrial, tecnotrónica, y sobre todo, de la información y la publicidad ${ }^{42}$.

Los años de este proceso democratizador, se encuentran sujeto a una profunda transformación de la prensa diaria no sólo en su estructura, sino en cuanto al tratamiento informativo. Cierres y nuevas aperturas de medios, nuevas ofertas informativas fruto de los permanentes cambios políticos, nuevos mercados publicitarios, etc.,... cuya reacción más inmediata es unas considerables dosis de desconfianza de los lectores hacia los medios que tradicionalmente venían siendo habituales. La ansiedad informativa del momento, cuestión que nos lleva a considerar el alto índice de lectura como una característica de la época, se desplaza también hacía nuevos diarios a fin de contrastar informaciones, o bien para buscar nuevas ofertas informativas más educadas a las necesidades vigentes, a la vez que tiene lugar una lenta pero progresiva penetración de los medios estatales (El País, Diario 16 fundamentalmente).

La transición trae consigo la necesidad de nuevas aventuras empresariales en materia de edición, una diversificación de las ofertas de comunicación y nuevas llamadas publicitarias al interés del lector. La competitividad en el mercado, se muestra en la necesidad permanente de abordar constantes

40 BRAOJOS GARRIDO, A., Prensa y «opinión pública» política en la Andalucía contemporánea, Facultad Ciencias de la Información-Ediciones Alfar, Sevilla, 1991.

${ }^{41}$ Así se anuncia la posible creación de una Facultad de Ciencias de la Información en Sevilla, a partir de un informe presentado por el Centro Español de Nuevas Profesiones que eleva ante el Rectorado de la Universidad hispalense. EL CORREO DE ANDALUCÍA, 25-V-1979.

${ }^{42}$ TIMOTEO ALVÁREZ, J., "Cambio de ciclo", en Historia de los medios de comunicación en España. Periodismo, imagen y publicidad (1900-1990), Barcelona, Ariel, 1989, p. 385. 
cambios para pervivir en un mercado de audiencias. La progresiva introducción de la informática y el diseño en el campo periodístico, los nuevos sistemas de impresión utilizando incluso color... comienzan a significar nuevos referentes tecnológicos que van a superar las viejas rotativas propias del anterior régimen. Cuestiones éstas, que obligan sobre la base de unas permanentes mejoras motivadas bajo finalidad competitiva.

Por otro lado, la apertura democrática introdujo nuevas formas y estilos de gobierno local, autonómico y estatal, que vinieron a ofrecer un impulso sustancial a la publicidad insertada en los medios impresos como elementos fundamentales para la llegada de determinados mensajes y servicios a la población. Cuestión decisiva si le añadimos, también la colaboración de entidades privadas o públicas. Aparece también, el uso político de la publicidad institucional, como fruto de esta importante inversión realizada, y en consecuencia, resulta ser un instrumento de presión directo desde organismos públicos sobre determinados medios.

Son tiempos de agilidad informativa en donde el comentario editorial y la información extra oficial toman mayor carta de naturaleza. Se desarrolla la información municipal, y sobre todo, las nuevas instituciones autonómicas, las cuales incluso en su etapa preautonómica se van abriendo paso, paulatinamente de ser noticia a conformar secciones informativas de carácter permanente. Aparecen las secciones de comentarios al margen y breves, cuya trascendencia e interés anecdótico sirven en la mayoría de los casos para intuir o argumentar los resultados de un hecho de una situación política pasada o próxima ${ }^{43}$.

Tras décadas de inmovilismo, la prensa diaria andaluza realiza una completa reacomodación ${ }^{44}$. Por lo que en coherencia con la nueva situación democrática, y la necesidad de estar informado al hilo de la velocidad con que se suceden los hechos, pasemos a analizar someramente algunas consideraciones generales alrededor de los numerosos títulos informativos ya aparecidos en una etapa de aperturismo post franquista y con un marcado acento regional. Ahora bien, la iniciativa empresarial en pro de nuevas cabeceras durante los años en estudio se centra casi prioritariamente en la ciudad hispalense, de tal forma que en el resto de provincias andaluzas, las influen-

\footnotetext{
${ }^{43}$ Este es el caso de las secciones Flash en ABC edición de Sevilla, El politicón en EL CORREO DE ANDALUCÍA, así como de El Satiricón en SUROESTE. La continuidad en el análisis de estas secciones nos descubre un interesante método para conocer cómo los rumores o los indicios se vuelven más tarde realidad.

${ }^{44}$ CHECA GODOY, A., Historia de la prensa andaluza, Sevilla, Fundación Blas Infante, 1.991, pp. 447 - 491.
} 
cias de la antigua prensa del Movimiento se hacen más patente en su diferentes grados de evolución. La consolidación del libre mercado da al traste con el objeto que da sentido a las Hojas del Lunes, las cuales en su ediciones incluso con otros nombres, con excepción hecha de Jaén y Huelva acaban por desaparecer, mientras que la prensa se consolida en su edición todos los días de la semana.

Si bien es cierto que el carácter definitorio de los medios andaluces durante el franquismo es el peso específico que en ellos juega el Estado, con excepciones en Cádiz y Sevilla, es cierto que una vez sucede el periodo de la transición, la importancia se polariza hacia sectores cercanos al socialismo en España. Así lo constatan Checa en su libro ya citado y Montabes Pereira, y el tema resulta lo suficientemente interesante como para analizarlo más con profundidad.

Resulta especialmente significativo por cuanto en tiempos de UCD, las centrales sindicales conocen por el I Congreso Nacional de UCD celebrado el 19 octubre de 1978 que el contenido de la ponencia programática de la coalición, daba por válido que el Estado no debía ser titular de ningún órgano de expresión escrito. UGT difunde reiteradamente que la maniobra de UCD respondía al interés que tenía ese partido por comprar, a bajo precio, los diarios más rentables, con el objeto de redondear una operación sibilina en la que se trata, al igual que en los tiempos del franquismo, de dominar desde el poder la televisión, la radio, las agencias y la prensa ${ }^{45}$. La presión de las fuerzas opositoras es tan virulenta que UCD pese a las acusaciones que recibe, sólo actúa acordando la suspensión en 1977 del semanario 7 Fechas y en junio de 1979, con el cierre de actividades de la agencia Pyresa y otros seis diarios más todos ellos deficitarios. Cualquier otra actuación en otro sentido hubiera dado al traste con el consenso necesario durante el periodo constituyente, y una vez superado éste, hubiera sido un factor de crisis política para el progresivamente deteriorado partido gubernamental. Si bien el deseo de acabar con las cabeceras se vuelve partidista en tiempos de UCD, no es menos cierto que el retraso en la decisión última del gobierno beneficio a los medios privados y facilitó a la oposición socialista, un tiempo decisivo para elaborar una estrategia de acceso a los medios de la Cadena, más significativos en cuanto a su cobertura, y con mayores posibilidades de ser reconducidos en

\footnotetext{
45 ARRIBA, 7-XI-1978. Citado en : ZALBIDEA BENGOA, B., “La prensa del Movimiento en los primeros años de la Transición (1975-1979)", op. cit, p. 490.
} 
cuanto a sus pérdidas. Espacio de tiempo por otro lado vital, para la consolidación en Andalucía y en todo el Estado en general. De una etapa informativa donde la supervivencia de los diarios depende de la adecuación a la demanda de los lectores, y no tanto por la opinión o a ayuda dictada desde el Estado. Espacio de tiempo trascendente a la espera de modificaciones en estos medios que contribuyó aún más a la pérdida de credibilidad y a la necesaria venta de estos medios.

Una modificación fundamental de esta etapa pre democrática la va a significar el hecho de que los medios, rompiendo su tradicional provincianismo, comienzan a abordar cada vez más la perspectiva de un tratamiento informativo de ámbito andaluz. La constitución de la Junta de Andalucía durante sus dos gobierno preautonómicos, va a contribuir poderosamente a ello. La aparición institucional del primer Gabinete de Prensa de la Junta de Andalucía va a empujar decisivamente en tal sentido, como actitud institucional antes los medios de comunicación, y cuyo ejemplo sería con posterioridad continuados por las primeras corporaciones locales democráticas $^{46}$.

En esta línea, si entendemos el agitado proceso autonómico andaluz como uno de los grandes núcleos informativos de Andalucía durante estos años, no cabe duda que la prensa ocupa un papel singular en la formación de un sentido autonomista. No sólo por cuanto el proceso para la llegada de la democracia se encuentra asociado a la vertebración del Estado en torno a la autonomías; sino por cuanto el papel concientizador, movilizador y reivindicativo que juega conforme al importante papel que juega Andalucía como única comunidad que utiliza dificultosa vía del artículo 151 de la Constitución para acceder a la autonomía. Por ende, importante también a la hora de evaluar sociológica y políticamente, con seguridad quizás los dos hitos más significativos de la transición de Andalucía: las multitudinarias manifestaciones del día 4 de diciembre de 1977 y la victoria política, que no jurídica, en el decisivo referéndum del 28 de febrero de $1980^{47}$. La conquista del Estatuto de Autonomía para Andalucía se convierte pues, en el gran

\footnotetext{
${ }^{46}$ Como Jefe del Gabinete de Prensa del primer gobierno preautonómico andaluz, se nombra a Antonio Burgos, Cfr. EL CORREO DE ANDALUCÍA, 6-VI-1978, aunque presenta su dimisión más tarde según anuncia ABC el 19-X-1978. Con posterioridad recae la responsabilidad en Dolores Cintado. Cfr. SUROESTE, 15-XI-1978.

${ }^{47}$ La portada de EL CORREO DE ANDALUCÍA el 4 de diciembre, titula expresivamente en grandes caracteres en negro sobre portada de fondo blanco: Hoy un día de bandera. Una primera página de la historia de Andalucía que debemos llenar todos con nuestra presencia.
} 
eje temático de la etapa que analizamos, y del que se desprende interesantes campos de estudio.

De forma paralela, los medios escritos andaluces de la transición se constituyen en vehículo fundamental para la recuperación y difusión de personajes, símbolos y hechos, en definitiva de parte de nuestra historia. Es más, las arbitrarias divisiones que se realizan durante el período franquista en aspectos como el militar, eclesiástico, judicial y universitario unidos a una rígida administración centralista ejercida desde Gobiernos Civiles y Diputaciones, hacen que Andalucía no exista como proyecto unitario entre 1936 y $1976^{48}$. A partir de un análisis economicista aparece el tratamiento de lo andaluz como realidad supraprovincial, cultural y política más tarde.

Como fruto de esta reflexión primero desde ámbitos económicos y más tarde literarios, surge la corriente historiográfica denominada Andalucismo Histórico, fundamentalmente centrado en el figura y obra de su principal promotor: Blas Infante Pérez, de su círculo de gentes y acción ${ }^{49}$. Tendencia investigadora que algunos autores por desconocimiento aún hoy identifican precipitadamente como justificación de determinada ideología política, sin valorar que nace al amparo de todas las ideologías y que bajo normalizado consenso, siendo institucionalizada mediante el reconocimiento que el futuro Parlamento andaluz hace de Blas Infante y los símbolos ${ }^{50}$.

Andalucía se reencuentra con una parte sustancial de su historia silenciada, si bien los estudios realizados en un primer momento tienen más un enfoque divulgativo que a una seria valoración de los hechos históricos en sí. La reflexión histórica sobre nuestro pasado viene incentivada por la asunción de una simbología autonómica (escudo, bandera, himno), en un contexto de precedentes hechos regionalistas (nacionalistas y/o autonomistas) pro-

${ }^{48}$ HIJANO DEL RÍO, M. y RUIZ ROMERO, M., «Justificaciones históricas en la génesis del Estatuto de Autonomía para Andalucía. 1.982», en Actas del I Congreso Internacional sobre la Historia de la Transición y Consolidación Democrática en España, Madrid, vol.I, Universidad Nacional de Educación a Distancia-Universidad Autónoma de Madrid, 1.995, pp. 555 - 565.

${ }^{49}$ Por acuerdo de todos los partidos en abril de 1.983, mediante Proposición no de Ley 6/83, se le nomina con el decimonónico título de Padre de la Patria Andaluza, en reconocimiento a su labor como «precursor de la lucha por la consecución del autogobierno que hoy representa el Estatuto de Autonomía», tal como obliga a constar en la introducción de dicha Ley Orgánica.

${ }^{50}$ Entendemos esta corriente historiográfica como la denominamos, al estudio científico de aquellas personalidades, doctrinas, hechos, evolución y problemática transcendentes dentro del pensamiento regionalista/nacionalista en el impulso en pro de la autonomía de la Andalucía contemporánea. En el contexto de los intentos de vertebración territorial y búsqueda de personalidad de España y sus comunidades. Sustancialmente coincidentes con la misma biografía de Infante (Cfr. INIESTA COULLAUT-VALERA, E., «Un primer censo de andalucistas históricos de la etapa infantiana», en Actas del III Congreso sobre el Andalucismo Histórico, Sevilla, Fundación Blas Infante, 1.989 , p. 360) 
cedente de la propia acción de Infante desde comienzos de siglo ${ }^{51}$. Pese a la fractura de los años franquistas, la recuperación de la autonomía se resuelve mediante ecos heredados y elementos nuevos. Los cuales, pese nacer fruto de un limitado círculo de nacionalistas, son asumidos con normalidad primero por la sociedad civil en la calle, y más tarde por las representaciones democráticas en instituciones. Entre otras cuestiones gracias al papel difusor popularizador que de ella hacen los medios, y que contribuyen a que el 4 de diciembre de 1977 sea el pueblo en manifestación quien legalice sus símbolos antes que las propias instituciones ${ }^{52}$.

Sin embargo Andalucía, salvos los intentos apuntados, durante la transición no consigue vertebrar un medio escrito de carácter regional, capaz de superar el rígido ámbito de influencia provincial, o al menos, de priorizar preferentemente noticias de ámbito andaluz.

La prensa andaluza de la transición contribuye también a la normalización de las pautas propias del comportamiento democrático. La diversificación de opiniones y editoriales, enriquecen al lector no sólo desde una perspectiva ideológica, sino que le preparan la madurez política de un ciudadano poco acostumbrado a ejercer su derecho a voto y al ejercicio de sus derechos. Así, durante las primeras campañas democráticas, aunque en diferente intensidad, los medios escritos se convierten en tribuna pública para el análisis de los diferentes programas y el seguimiento de los actos diarios de las respectivas campañas. Se inician las entrevistas a fondo de los candidatos y se confrontan posturas ante determinados problemas. Más que dirigir al lector, por la fidelidad de éste al medio, se le ofrece una panorama de conjunto abierta a diferentes opciones electorales. La polaridad ideológica de los medios de comunicación que hoy existe, resulta impensable en aquellas fechas. Especialmente durante el proceso constituyente y las primeras elecciones generales y municipales, donde los análisis de extrapolación de los resultados de las constituyentes son constantes, y comienzan las primeras encuestas y la extrapolación de datos con las primeras consultas. Se incorporan secciones específicas para tratar los problemas y la cultura de Andalucía, la información sindical se consolida junto a la económica, y de igual

${ }^{51}$ La Ley del Parlamento de Andalucía 3/1982 de 21 de diciembre sobre el Himno y el Escudo de Andalucía y el propio Estatuto de Autonomía, en referencia a la bandera, sitúan sus orígenes a la Asamblea de Ronda de 1918.

${ }^{52}$ Puede consultarse todo los editado sobre este aspecto en las trescientas cincuenta referencias apuntadas en : HIJANO DEL RÍO, M. y RUIZ ROMERO, M., «Bibliografía sobre el Andalucismo Histórico», en Boletín del Centro de Estudios Históricos de Andalucía, (6), 1.996, pp. 4 - 20. 
forma, se realizan explicaciones al lector para el ejercicio de su derecho al voto. Gran parte de los medios y los profesionales de la información en Andalucía se vuelven escuelas de ciudadanía en un importante sector de población.

Sin embargo, existen casos en donde se demuestra que tanto la propia capacidad de los medios a la hora de evaluar la dimensión real una vez se realiza el seguimiento de una noticia, y que por otro lado, nos ofrece una idea de queda superada por la realidad jurídica de un Estado que avanza a pasos de gigante $^{53}$.

En resumen, el análisis de la prensa andaluza durante el periodo de la transición nos lleva a considerar la etapa en general en un sentido diacrónico y en constante evolución. La realidad andaluza si bien no resulta homogénea, si es paralela, por cuanto viene marcada sobre la década de los años setenta, con una fuerte implantación de medios de la Cadena del Movimiento, si bien estos pierden intensidad en audiencia en la medida que pasan los años. Los medios consolidados de carácter independiente respecto a la órbita franquista pero imbuido de la realidad del régimen, se incorporan con normalidad a la defensa del proceso democrático y autonómico. Realizando un esfuerzo amplio por introducir en sus páginas nuevas temáticas hasta entonces desconocidas, acordes con su nueva línea editorial abiertamente comprometida en defensa de la democracia y en el rechazo de tendencias extremistas, violentas o reaccionarias. Son nulos los elementos de crispación o enfrentamiento entre diarios en estos momentos. En un principio viene marcada por un el índice de penetración de la prensa estatal, si bien es bajo al comienzo de la transición, aumenta una vez los procesos democráticos se consolidan. La aparición de nuevos medios, fruto del alto interés informativo, provoca la creación de nuevas audiencias que se incorporan al mercado de la prensa diaria en sus diferentes vertientes: vespertinos, prensa política, revistas... Resulta una época de gran iniciativa empresarial, si bien ésta no

\footnotetext{
53 Así, el diario ABC en referencia al momento de la aprobación por parte del Consejo de Ministros el día ocho, del Real Decreto por el que se aprueban los Estatutos de la Mancomunidad General de Diputaciones Provinciales de Régimen Común (RD 169/1977) expresa en portada y en páginas interiores una visión un tanto apologética en cuanto las posibilidades reales de la norma en sí misma y al respecto del caso andaluz: Consejo de Ministros. Aprobada la Mancomunidad de Diputaciones de Andalucía. ABC, 9-II-1977. Curiosamente esta mismo situación se repite más tarde, cuando el primer Gobierno ucedista presenta su oferta preautonómica a la Asamblea de Parlamentario de Andalucía. En esta ocasión, EL CORREO DE ANDALUCÍA, 7-I-1978, con grandes alardes tipográficos confunde al lector al hacerle creer que el Decreto-Ley de creación del nuevo ente había sido aprobado cuando en realidad se trata de una consulta previa que se realiza, la cual es rechazada desde el órgano colegiado andaluz de representantes.
} 
llega a concretarse pese al intento, en la consolidación de un medio de ámbito andaluz.

Pero lo que es más importante, las matizaciones particulares que dan riqueza a esta síntesis general están aún por llegar. Los cambios profundos habidos en la prensa y en la sociedad andaluza justifican continuar con la tarea de profundizar en nuestra historia reciente. De tal forma que ante la falta de archivos oficiales sobre la transición, salvo honrosas excepciones, el estudio de la prensa andaluza como fuente y como documento histórico constituye un elemento de análisis esencial. La conservación, estudio y difusión de este rico patrimonio documental nos compromete a todos. Ello hemos intentado apuntar en estas breves líneas.

\section{Anexo \\ Creación de una Hemeroteca General de Andalucía}

Proposición No de Ley en Pleno aprobada por el Parlamento en sesión ordinaria de los días 28, 29 y 30 de marzo de 1995 (4 - 94 / PNLP - 05108)

$1^{\circ}$.- Instar al Consejo de Gobierno para que establezca convenios de colaboración con las instituciones, diputaciones provinciales, ayuntamientos y particulares que permitan conocer, estudiar y reproducir, dentro de los márgenes legales para un depósito central, los fondos documentales referidos a la prensa escrita existente en Andalucía, así como otros fondos que pudieran existir fuera de nuestra Comunidad, muy especialmente en la Hemeroteca Nacional.

$2^{\circ}$.- Instar al Consejo de Gobierno a que, una vez realizado este trabajo, se cree la Hemeroteca General de Andalucía, vinculada a la Biblioteca de Andalucía. 